\title{
Examen d'analyses récentes portant sur l'Étude canadienne sur l'incidence des signalements de cas de violence et de négligence envers les enfants $(\mathrm{ECI})$
}

\author{
D. Potter, M.A.; T. Nasserie, M. Sc.; L. Tonmyr, Ph. D.
}

Cet article a fait l'objet d'une évaluation par les pairs.

Diffuser cet article sur Twitter

\section{Résumé}

Introduction : Cette analyse a comme objectif de rassembler les articles évalués par les pairs utilisant des données tirées de l'Étude canadienne sur l'incidence des signalements de cas de violence et de négligence envers les enfants (ECI) publiées depuis novembre 2011 ainsi que des suréchantillons provinciaux de l'ECI, d'évaluer la qualité de leurs conclusions, de les synthétiser et de faire le bilan des variations dans l'utilisation de ces données.

Méthodologie : Nous avons sélectionné les articles au moyen du suivi des demandes d'accès aux données de l'ECI effectué par l'Agence de la santé publique du Canada et au suivi des publications produites au moyen de ces données. Deux évaluateurs au moins ont examiné de façon indépendante les articles et en ont évalué la qualité.

Résultats : Nous avons conservé 32 articles. Leurs points forts étaient en général les suivants : objectifs de recherche clairement établis, variables de contrôle appropriées, analyses pertinentes, taille de l'échantillon suffisante, conclusions pertinentes et utilité pour les pratiques et politiques. Leur point problématique était le plus souvent une définition floue des variables et des critères d'inclusion des cas. La plupart du temps, les articles mesuraient les associations entre les caractéristiques des mauvais traitements et celles des enfants, des pourvoyeurs de soins, du ménage et de l'organisme ou de la personne à l'origine du signalement et ils s'intéressaient aux conséquences des signalements, par exemple l'ouverture d'un dossier pour une prestation continue de services ou le placement.

Conclusion : Les articles utilisant des données de l'ECI ont obtenu des scores positifs pour la plupart des indicateurs de qualité. Dans leurs analyses les plus récentes, les chercheurs se sont concentrés sur les catégories de maltraitance insuffisamment étudiées jusqu'ici (exposition à la violence conjugale, négligence et violence psychologique) et ont étudié les facteurs spécifiques touchant les enfants des Premières Nations. Les données des suréchantillons de l'ECI ont été sous-utilisées. L'utilisation de techniques d'analyse multivariée a augmenté.

Mots-clés : violence à l'égard des enfants, maltraitance des enfants, surveillance de la santé publique, épidémiologie

\section{Introduction}

L'Étude canadienne sur l'incidence des signalements de cas de violence et de négligence envers les enfants (ECI) a été réalisée pour trois cycles $\left(1998^{1}, 2003^{2}\right.$ et $\left.2008^{3}\right)$ et a fourni de précieux renseignements au sujet des enquêtes sur les cas signalés de violence et de négligence envers les enfants à l'échelle du pays ${ }^{4}$. Les données de l'ECI portent sur les enfants dont la situation a été signalée à un organisme d'aide à l'enfance

\section{Principales constatations}

- Parmi les 32 articles publiés évalués par des pairs dans le cadre de notre analyse, la plupart était de grande qualité, avec des objectifs de recherche clairement établis, des variables de contrôle et des méthodes d'analyse pertinentes, une taille d'échantillon suffisante, des conclusions pertinentes et une utilité dans le champ des pratiques et politiques.

- Les chercheurs utilisant des données de l'ECI ont surtout travaillé sur les catégories de maltraitance insuffisamment étudiées jusqu'ici, en particulier l'exposition à la violence conjugale, la négligence et la violence psychologique, et ils ont également étudié les facteurs spécifiques touchant les enfants des Premières Nations.

- L'utilisation de techniques d'analyse multivariée a augmenté.

en raison d'allégations de maltraitance ${ }^{1-3}$. Elles constituent une source d'information sur les enfants qui ont subi de la négligence (alléguée ou corroborée), qui ont été exposés à de la violence conjugale et qui ont subi de la violence psychologique, physique ou sexuelle. Elles portent sur les caractéristiques de l'enfant, celles du pourvoyeur de soins, celles du ménage et celles de l'organisme chargé de l'enquête ainsi que sur les résultats à court terme des services fournis, par exemple le placement. Les données de l'ECI sont utilisées par les décideurs de niveau supérieur des organismes d'aide à l'enfance afin de décider de l'attribution des ressources, de cibler les populations à risque, de connaître les tendances dans

Rattachement des auteurs :

Division de la surveillance et de l'épidémiologie, Agence de la santé publique du Canada, Ottawa (Ontario), Canada

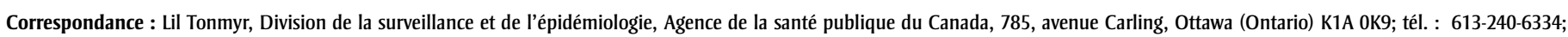
téléc. : 613-941-6439; courriel : Lil.Tonmyr@phac-aspc.gc.ca 
le signalement des mauvais traitements, de valider les conclusions de chaque organisme et d'influencer directement les pratiques ${ }^{4}$. Ces données sont également utilisées par un large éventail d'experts en médecine clinique, en santé publique, en services sociaux, en droit et justice, en éducation et en sports et loisirs ainsi que par des groupes confessionnels ${ }^{5}$.

ECI 2008 : Données principales ${ }^{3}$ décrit l'étendue et la portée de cette enquête. Chaque cycle de l'ECI contient de l'information sur un grand échantillon de cas de maltraitance signalés aux organismes d'aide à l'enfance et faisant l'objet d'une enquête. Les intervenants en protection de l'enfance de certains organismes au Canada ont répondu à l'ECI pour chaque enquête réalisée pendant la période trimestrielle de collecte de données à l'automne de l'année de l'étude. Les définitions des diverses catégories de maltraitance ont été fournies aux intervenants participant afin d'assurer l'uniformité des données. On a utilisé une méthode d'échantillonnage stratifié en grappe incluant les principaux organismes et les organismes des Premières Nations et portant sur toutes les provinces et tous les territoires. Certaines provinces et certains territoires ont versé des fonds supplémentaires à l'ECI afin d'obtenir des données les concernant spécifiquement (suréchantillonnage).

L'ECI 2008 diffère des cycles de 1998 et de 2003 en ce que le suivi des enquêtes sur le risque de futurs mauvais traitements a été effectué séparément de celui sur les enquêtes d'allégations des différents mauvais traitements. De plus, les définitions des catégories de maltraitance ont évolué au fil du temps. Par exemple, l'ECI 2008 fait le suivi de trois sous-catégories d'exposition à la violence conjugale : exposition indirecte à la violence physique, exposition directe à la violence physique et exposition à la violence psychologique $^{6}$. Dans les cycles précédents, l'ECI considérait la violence conjugale comme une sous-catégorie de violence psychologique. Les différences entre les lois provinciales et territoriales et les modifications apportées au fil du temps aux procédures de détection, de signalement et d'enquête ont également une incidence sur les conclusions de l'ECI, ce qui signifie que les changements observés ne reflètent pas forcément des changements réels dans l'occurrence des mauvais traitements.
Un examen antérieur ${ }^{7}$ a résumé et critiqué 37 analyses évaluées par des pairs utilisant des données de l'ECI et publiées avant novembre 2011. Dans près de la moitié des cas, les analyses étaient descriptives, et pour les autres, multivariées. L'évaluation de la qualité des articles a permis de conclure que les articles fondés sur une analyse multivariée étaient généralement de meilleure qualité, fournissant des données probantes solides mieux en lien avec leurs objectifs que les articles fondés sur une analyse descriptive. Cet examen a recommandé pour la recherche à venir l'utilisation d'hypothèses directionnelles et de techniques éprouvées d'analyse multivariée ${ }^{7}$.

L'Agence de la santé publique du Canada ayant décidé de ne pas recueillir de données en 2013, notre examen fournit l'occasion de mettre à jour les conclusions de l'examen précédent et de se questionner sur la pertinence de l'ECI dans le champ des pratiques et des politiques. Seuls trois articles dans ce premier examen ${ }^{7}$ utilisaient des données de l’ECI 2008. De plus, les renseignements étaient limités puisque les données venaient tout juste d'être publiées. Par ailleurs, cet examen avait été critiqué pour avoir exclu les résultats touchant les suréchantillons des provinces. Depuis, un plus grand nombre d'articles utilisant ces données et des données des cycles précédents ont été publiés. Notre examen met donc l'accent sur les documents de la littérature reliée à l'ECI publiés après novembre 2011 ainsi que sur les analyses des suréchantillons de l'ECI indépendamment de leur date de publication.

Les utilisateurs de connaissances (décideurs et responsables des politiques) considèrent que la synthèse de recherches leur est utile. Les rapports de surveillance découlant de l'ECI ont ainsi été mentionnés comme étant un moyen d'obtenir rapidement de l'information $^{4}$. Nous espérons donc que notre examen sera utilisé comme tel sur les thèmes que les données de l'ECI permettent de traiter ainsi que pour la qualité des articles auxquels il fait référence. Notre examen est également susceptible d'encourager les chercheurs à approfondir les connaissances sur la maltraitance et sur les interventions des organismes d'aide à l'enfance à cet égard. Il est également susceptible de servir à améliorer l'ECI en tant qu'outil de surveillance, grâce à l'identification de certaines lacunes dans le processus de collecte et d'analyse des données. Enfin, il fournit des données susceptibles d'accroître le niveau de sensibilisation à cette importante question de santé publique.

Plus spécifiquement, les objectifs de notre examen étaient les suivants :

- compiler et récupérer toutes les études évaluées par les pairs et utilisant les données de l'ECI publiées depuis novembre 2011 et les données des suréchantillons provinciaux et territoriaux de l'ECI recueillies depuis la création de l'étude;

- évaluer la qualité de ces études;

- résumer les conclusions de ces études;

- caractériser les évolutions dans l'utilisation des données de l'ECI.

\section{Méthodologie}

Nous avons sélectionné les recherches originales publiées dans des revues à comité de lecture, faisant l'hypothèse que celles-ci seraient de bonne qualité et comprendraient suffisamment de renseignements sur les méthodes et les analyses pour assurer cette qualité. Les autres sources, essentiellement les chapitres d'ouvrage et les présentations, ne respectent pas toujours une présentation normalisée de l'information ou ne contiennent pas d'objectifs ou d'hypothèses à tester. Notre outil d'évaluation de la qualité n'aurait donc pas été approprié. De plus, l'examen se limitant aux articles de revues à comité de lecture, les lecteurs intéressés pourront localiser facilement nos sources primaires et y avoir accès. Le tableau 1 présente la liste des cycles de l'ECI pour l'ensemble du Canada, pour les provinces ou territoires ayant réalisé un suréchantillon et pour les Premières Nations.

Les articles ont été sélectionnés à partir du suivi par l'Agence de la santé publique du Canada des demandes d'accès aux données de l'ECI. Dans un souci d'exhaustivité, nous avons également recherché les articles publiés par les auteurs de l'équipe de recherche de l'ECI, ces derniers n'ayant pas eu besoin d'obtenir d'autorisation pour accéder aux données de l'ECI et leurs publications ne figurant par conséquent pas dans les demandes d'accès.

La figure 1 synthétise la stratégie de sélection des articles (source, critères d'inclusion 
TABLEAU 1

Cycles et suréchantillons de I'ECI et études des Premières Nations

\begin{tabular}{ll} 
Population & \multicolumn{1}{c}{ Cycles } \\
\hline Canada & $1998,2003,2008$ \\
Colombie-Britannique & 1998,2008 \\
Alberta & $2003,2008,2013$ \\
Saskatchewan & 2008 \\
Ontario & $1993,1998,2003,2008,2013$ \\
Québec & $1998,2008,2014$ \\
Territoires du Nord-Ouest & 2003 \\
Premières Nations & $1998,2003,2008$ \\
\hline
\end{tabular}

et d'exclusion) et présente la liste des questions visant à évaluer leur qualité. Nous avons repris les mêmes questions que dans le premier examen des données de l'ECI ${ }^{7}$. Chaque article a été examiné de façon indépendante par deux évaluateurs pour en confirmer l'inclusion et en évaluer la qualité. Les écarts de cotation ont fait l'objet de discussions entre les évaluateurs jusqu'à obtention d'un consensus, ou de discussions avec un troisième évaluateur. Les évaluateurs n'ont pas examiné les articles qu'ils avaient eux-mêmes rédigés.

\section{Résultats}

Sur les 32 études sélectionnées, 20 reposaient sur une analyse multivariée contre 12 sur une analyse descriptive, 24 utilisaient des données nationales contre 8 des données provinciales ou territoriales ( 5 sur le Québec et 3 sur l'Ontario) et une seule était consacrée aux données de l’ECI 2008 sur les Premières Nations.

Le tableau 2 décrit les objectifs de chaque étude et la méthodologie employée et présente les résultats de l'évaluation de sa qualité. Si $91 \%$ des auteurs ont décrit clairement les objectifs de leur recherche, seulement $40 \%$ ont fourni une définition claire de toutes les variables. Les critères d'inclusion des cas ont été présentés dans $72 \%$ des études et les méthodes d'échantillonnage étaient claires et pertinentes également dans $72 \%$ d'entre elles. Ont été considérées comme pertinentes à l'égard des questions de recherche des auteurs $78 \%$ des analyses. Le nombre de participants a été jugé suffisant $91 \%$ du temps. De manière générale, les procédures d'analyse et les résultats ont été clairement expliqués (72 \%) et présentés (75\%). Les conclusions ont été considérées comme pertinentes dans $81 \%$ des cas et toutes les études sauf une avaient des retombées claires dans le champ des pratiques ou des politiques.

Les objectifs de $28 \%$ des articles portaient sur des catégories spécifiques de maltraitance. Deux traitaient de la négligence, trois de la violence psychologique et deux de l'exposition à la violence conjugale Une étude portait sur l'exposition à la violence conjugale, les brutalités physiques ou la négligence comme uniques raisons de l'enquête. Aucune étude ne ciblait exclusivement la violence physique. Un rapport se penchait sur la fréquence des enquêtes conjointes entre les services de police et les organismes d'aide à l'enfance sur les cas de violence sexuelle par rapport à celles sur les autres formes de maltraitance.

Le tableau 3 synthétise les associations entre variables indépendantes, variables dépendantes et variables de contrôle dans les articles offrant des analyses multivariées. Dans cet examen, une analyse multivariée a été définie comme une analyse portant simultanément sur plusieurs variables, indépendantes ou dépendantes. Pour les articles décrivant plusieurs modèles, seul le modèle final a été inclus pour chaque variable dépendante testée.

Les variables indépendantes et les variables de contrôle ont été regroupées en cinq catégories : les caractéristiques des mauvais traitements, celles de l'enfant, celles du pourvoyeur de soins, celles du ménage et celles de l'organisme ou du signalement. Un article utilisant des techniques d'analyse multivariée n’a pas été inclus dans le tableau car ses analyses prenaient la forme d'arbres de classification et de régression ne pouvant être facilement synthétisés dans un tableau.

La plupart des analyses utilisant des catégories de maltraitance (violence physique, violence sexuelle, négligence, violence psychologique et exposition à la violence conjugale) à titre de variables indépendantes incluaient plus d'une de ces catégories. Une étude incluait seulement des variables de maltraitance correspondant aux différents types d'exposition à la violence conjugale, une autre incluait seulement la violence physique et deux incluaient seulement la négligence. De plus, deux études tenaient compte de la violence psychologique uniquement à titre de variable dépendante.

Les chercheurs ont utilisé fréquemment l'âge et le sexe de l'enfant comme variables indépendantes ou de contrôle concernant l'enfant. Ils ont eu tendance à utiliser dans leurs modèles certaines variables associées aux problèmes de fonctionnement chez l'enfant (mais pas toutes) et les ont parfois regroupés pour créer une variable dichotomique visant à illustrer la présence d'un problème quel qu'il soit. Ces problèmes de fonctionnement chez l'enfant étaient de nature physique, mentale, comportementale ou de développement. Les variables les plus fréquemment utilisées à propos des pourvoyeurs de soins étaient celles concernant leurs facteurs de risque, particulièrement la toxicomanie, les problèmes de santé mentale et l'absence de soutien social. Les variables les plus fréquemment utilisées pour les ménages étaient les dangers domestiques, un logement surpeuplé, les fréquents manques d'argent et les déménagements fréquents. Enfin, les variables les plus utilisées pour les organismes et le signalement étaient l'ouverture d'un ou de plusieurs dossiers et le signalement d'un cas par la mère ou une personne de la famille.

La catégorie de variables dépendantes la plus couramment étudiée (utilisée dans six articles) était l'ouverture d'un dossier pour services continus. Les chercheurs ont mesuré les associations entre cette variable et diverses variables indépendantes et de contrôle liées aux mauvais traitements et aux caractéristiques de l'enfant, du pourvoyeur de soins, du ménage et de l'organisme ou du 
FIGURE 1

Stratégie de recherche et évaluation des articles

a) Stratégie de recherche pour les articles contenant des analyses des données de l'ECI et des suréchantillons de l'ECI

\section{STRATÉGIE DE RECHERCHE}

Sélectionner les articles à partir des demandes d'accès aux données de I'ASPC

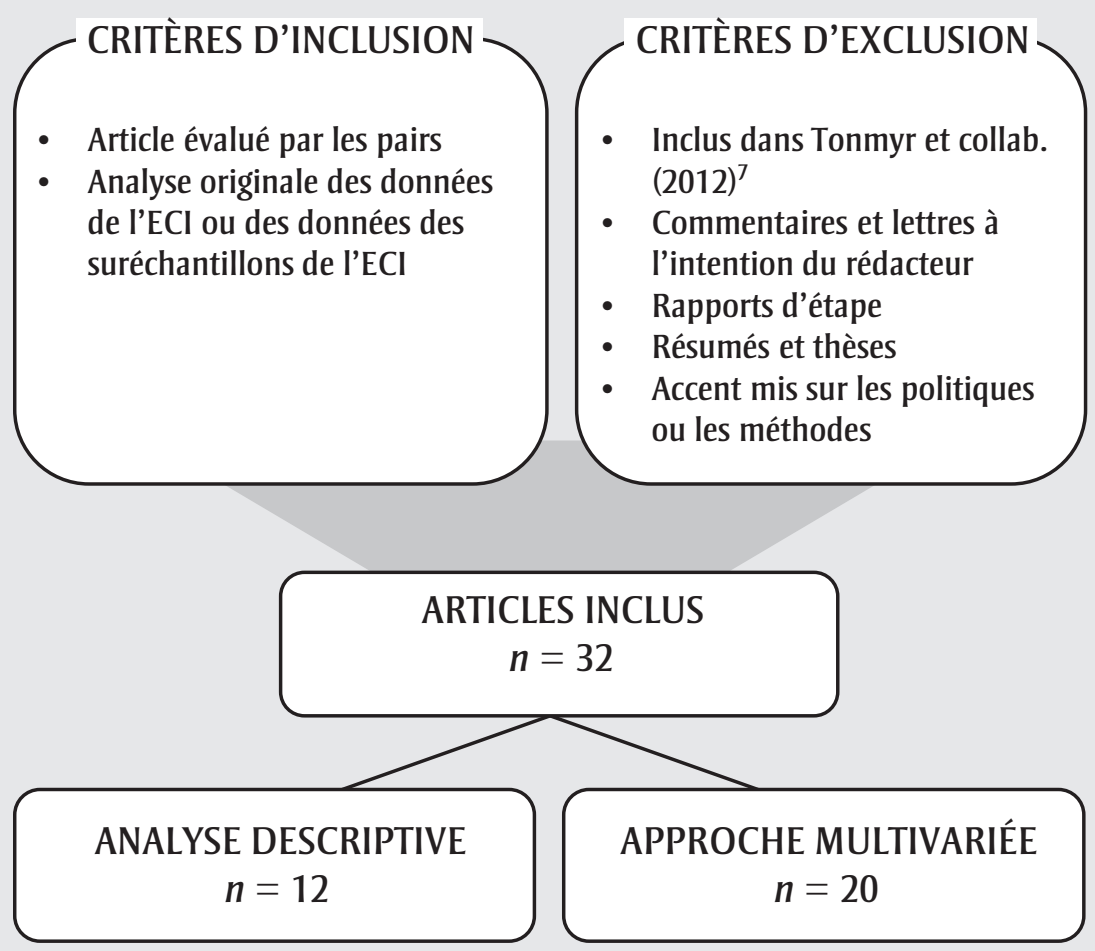

b) Questions d'évaluation critique

Thème

1. Quelle est la question de recherche ?

2. Les objectifs de la recherche sont-ils clairs ?

Méthodologie

3. Les variables utilisées sont-elles définies clairement ?

4. Les critères d'inclusion et d'exclusion sont-ils clairement mentionnés ?

5. La stratégie d'échantillonnage utilisée dans l'étude est-elle claire et pertinente ?

6. Les analyses utilisées pour répondre à la question de recherche sont-elles pertinentes?

7. Le nombre de participants est-il suffisant ?

Constatations et conclusions

8. La méthode d'analyse des données est-elle claire ?

9. Les résultats sont-ils clairement présentés ?

10. Les chercheurs tiennent-ils compte des facteurs de confusion possibles ?

11. Les conclusions s'appuient-elles sur les résultats de l'étude ?

12. Le thème de l'étude est-il utile pour le champ des pratiques et politiques ?

signalement. La deuxième catégorie de variables la plus couramment étudiée (utilisée dans quatre articles) était le placement. Ces articles incluaient de nombreuses variables relatives aux caractéristiques des mauvais traitements, à celles de l'enfant, à celles du pourvoyeur de soins, à celles du ménage et à celles de l'organisme ou du signalement.
Toutefois, seuls deux de ces quatre articles portaient sur les facteurs de risque liés aux pourvoyeurs de soins, et ils utilisaient un nombre limité de variables liées à cette catégorie. Les autres variables dépendantes étudiées (dans trois articles ou moins) étaient les cas de signalement retenus concernant les enfants autochtones, les mauvais traitements corroborés, les risques corroborés de mauvais traitements, de violence psychologique et de danger pour l'enfant et les variables liées aux problèmes de fonctionnement chez l'enfant (problèmes d'apprentissage et de développement, incapacités physiques et problèmes de santé, problèmes psychologiques et émotionnels, troubles d'intériorisation, troubles d'extériorisation, déficiences fonctionnelles) et enfin la participation des services de police aux enquêtes.

\section{Analyse}

Notre examen résume les résultats de 32 articles de revue à comité de lecture portant sur la qualité et les liens entre variables utilisant des données de l'ECI et publiés depuis novembre 2011 ou des données des suréchantillons de l'ECI. La majorité des articles évalués avaient des objectifs de recherche clairement établis, utilisaient des variables de contrôle valides (analyses multivariées plutôt que descriptives), réalisaient des analyses pertinentes au moyen d'échantillons de taille suffisante, tiraient des conclusions pertinentes et étaient utiles dans le champ des pratiques et politiques.

La majorité des études ne définissaient pas clairement les variables ou ne fournissaient pas de justification de leur inclusion aux analyses. Comme beaucoup incluaient un grand nombre de variables, il est probable que ce soit dû à la limite de mots imposée par les éditeurs plutôt qu'à un réel manque de justification de la part des chercheurs. Les critères d'inclusion et les méthodes d'échantillonnage ont été jugés peu clairs souvent lorsque les auteurs n'avaient pas expliqué comment ils avaient choisi les cas inclus dans leur analyse à partir de l'échantillon complet de l'ECI et avaient fourni un simple aperçu des méthodes d'échantillonnage de l'ECI. Le nombre de participants a été jugé insuffisant lorsque les chercheurs avaient sélectionné pour leur analyse des suréchantillons de cas de l'ECI de petite taille. L'une 


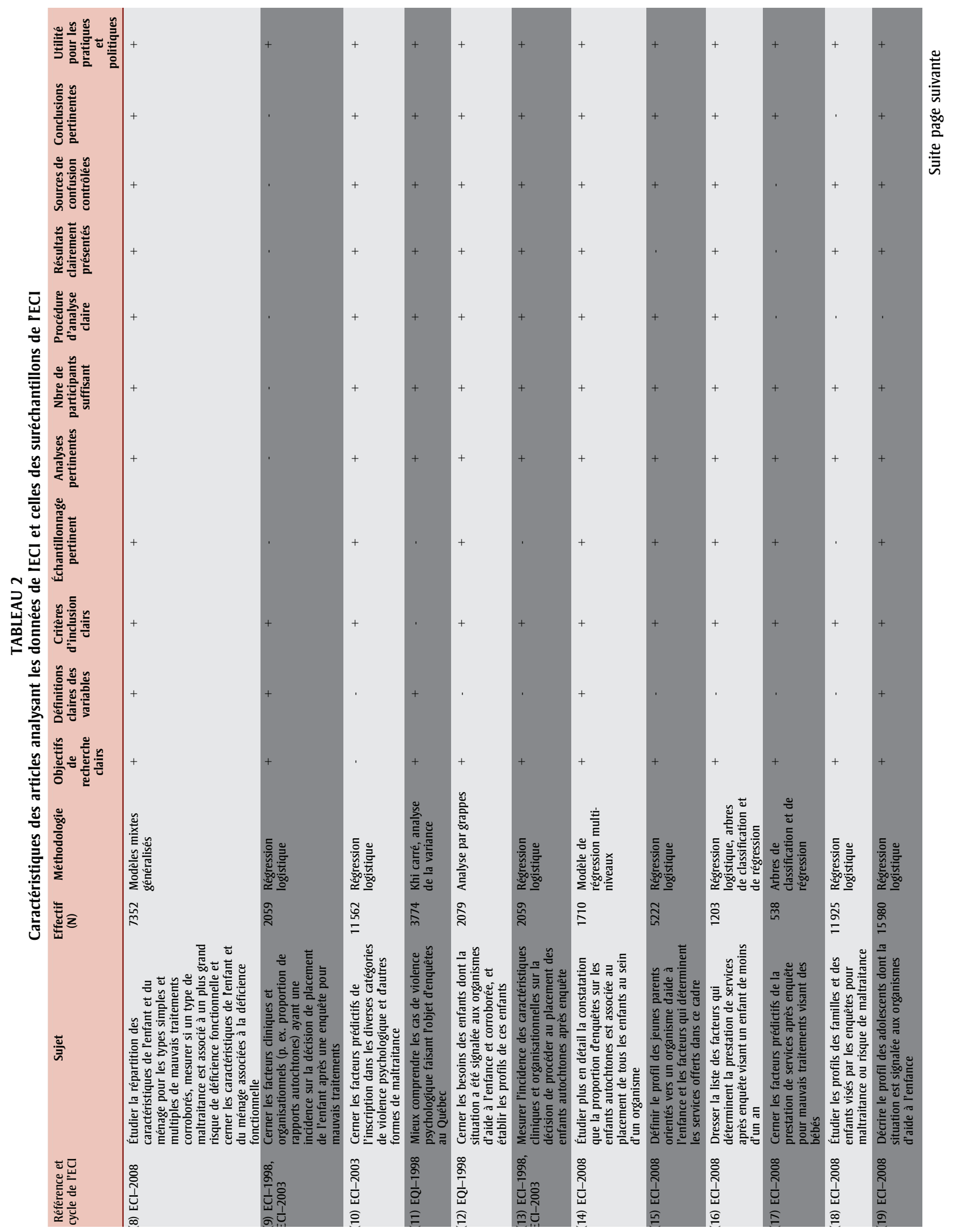




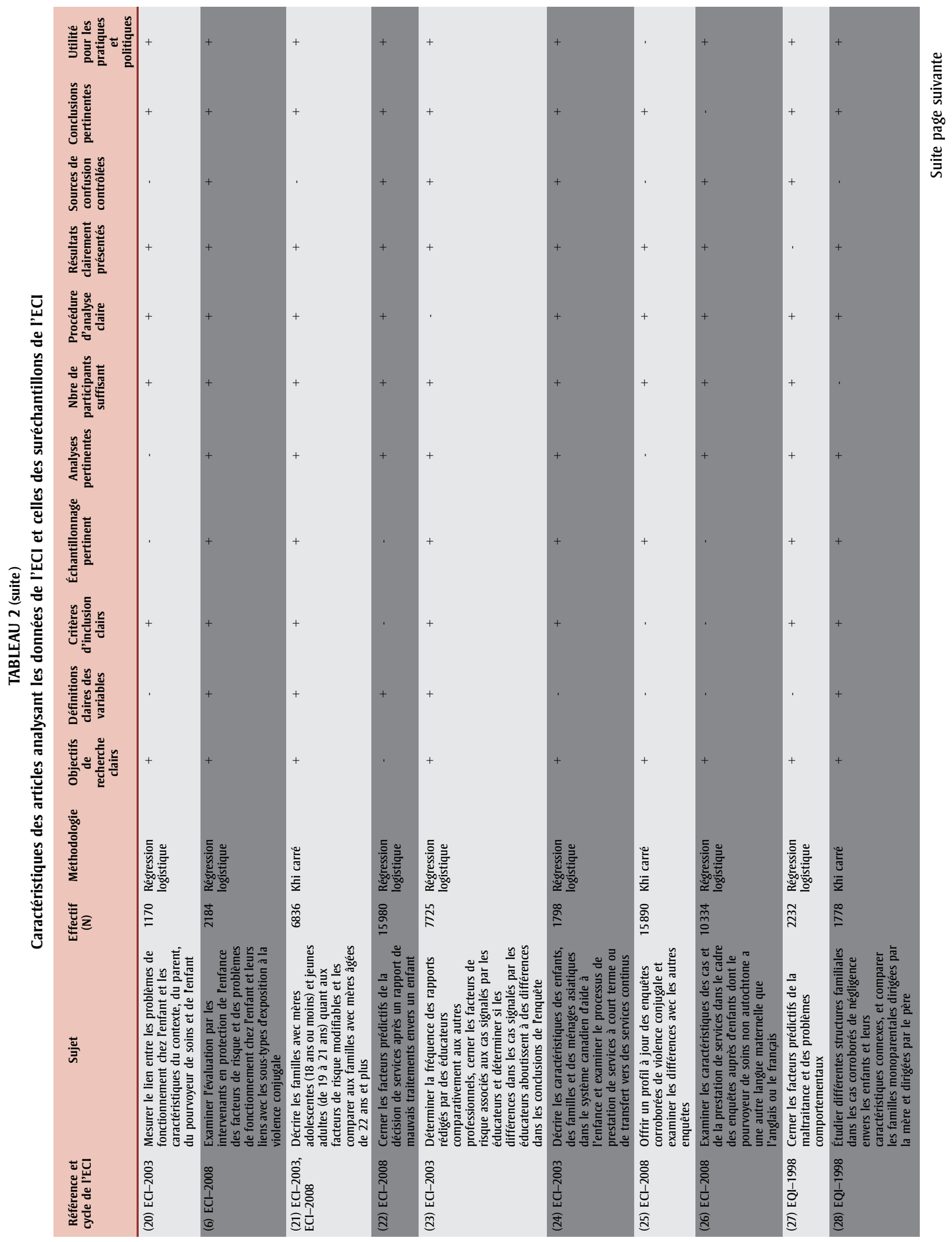




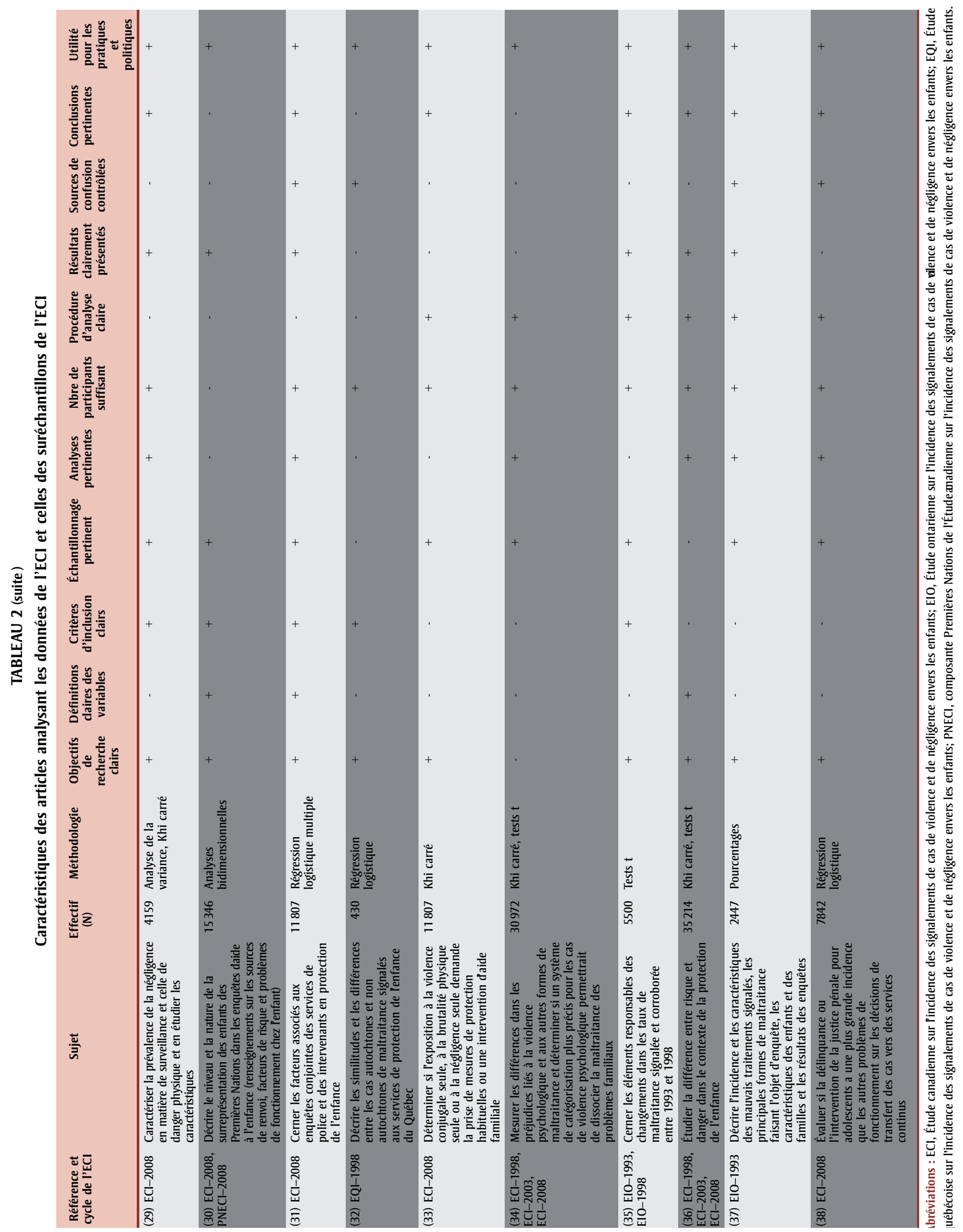


TABLEAU 3

Liens entre les variables indépendantes, dépendantes et de contrôle dans les articles présentant des analyses multivariable

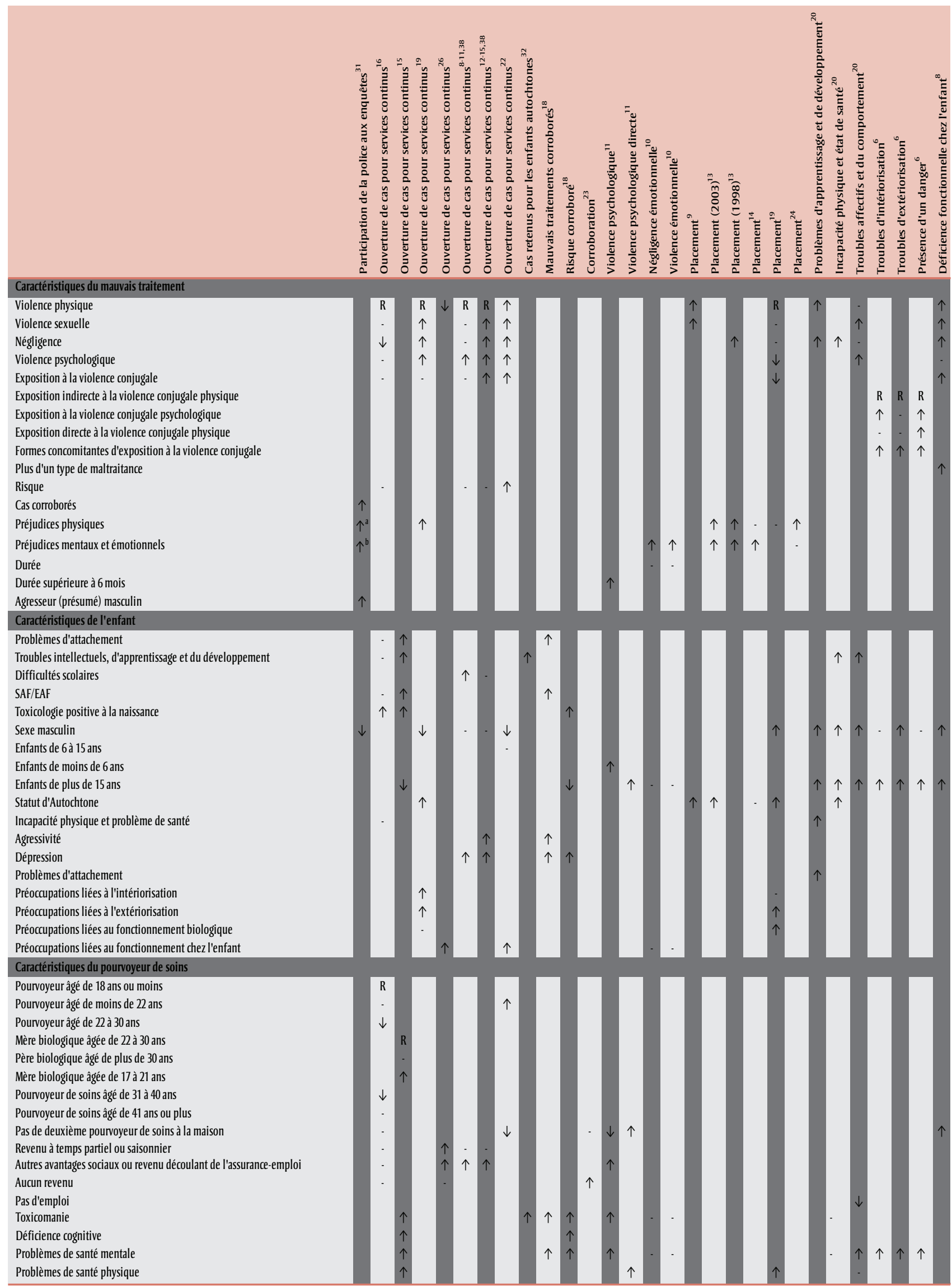

Suite page suivante 
TABLEAU 3 (suite)

Liens entre les variables indépendantes, dépendantes et de contrôle dans les articles présentant des analyses multivariable

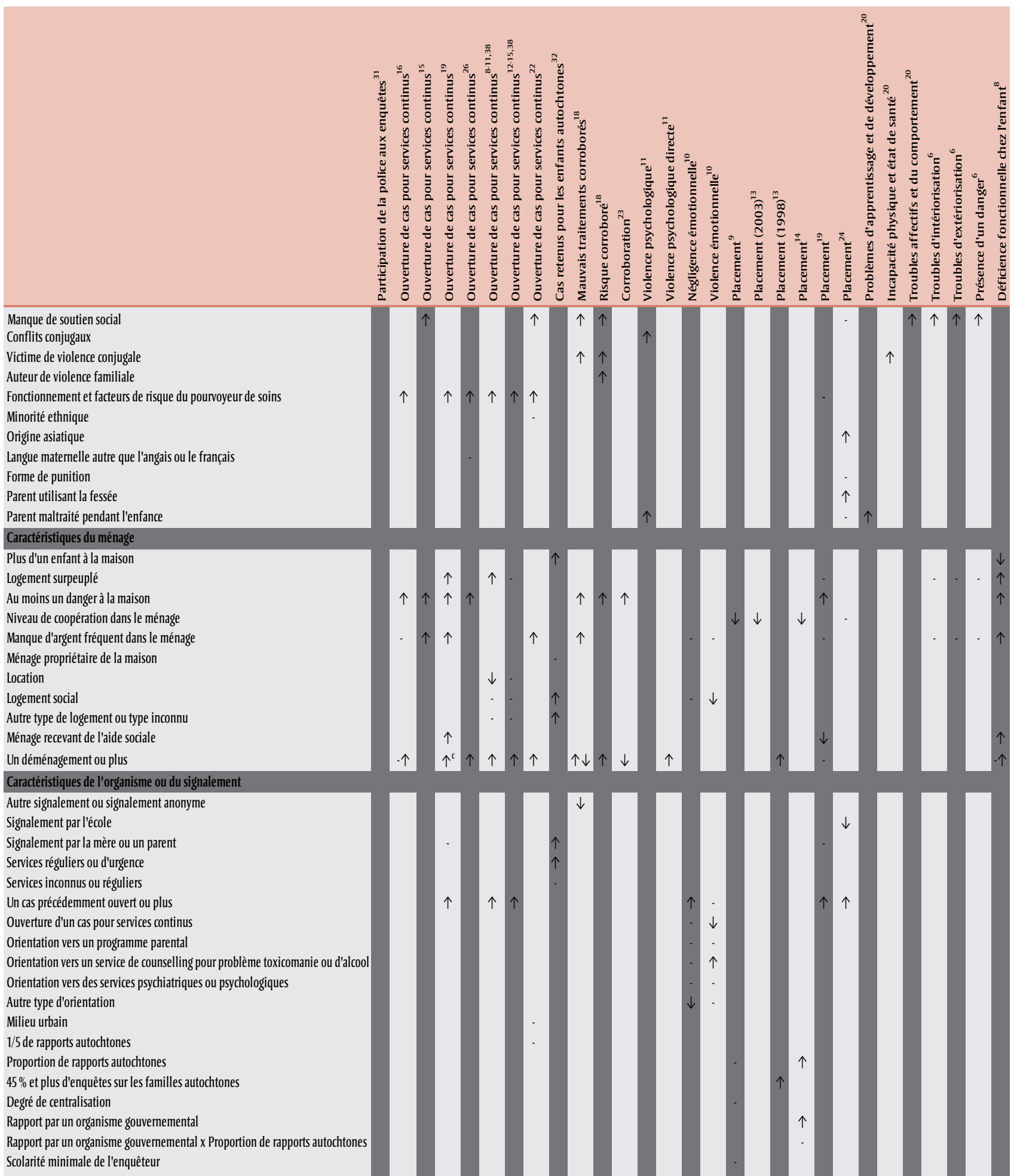

Abréviations : EAF, effets de l'alcoolisme foetal; $R$, groupe de référence; SAF, syndrome dalcoolisation foetale; -, pas de lien statistiquement significatif $(p>0,05)$; $\downarrow$, lien négatif statistiquement significatif ( $p<0,05)$; $\uparrow$, lien positif statistiquement significatif $(p<0,05)$.

Remarque : L'apparition de plus d'un symbole dans une cellule signifie que l'article présentait des catégories plus fines de la variable qui ont été combinées par souci de concision. Ces catégories avaient en outre des liens de différents ordres avec la variable dépendante.

${ }^{a}$ Cette variable combinait des dangers physiques et émotionnels.

${ }^{\mathrm{b}}$ Cette variable combinait des dangers physiques et émotionnels.

c La valeur de la variable était de deux déménagements ou plus. 
des forces de l'ECI est que l'échantillon est de taille suffisamment importante pour que soient menées des analyses multivariées complexes, ce qui explique que cet indicateur de qualité ait presque toujours reçu une note positive. Les analyses ont très souvent été jugées pertinentes et les résultats clairement présentés, sauf quand les auteurs n'avaient pas décrit quelles analyses ils avaient effectuées ou n'avaient pas clairement présenté leurs tableaux. Lorsque les analyses n'ont pas été jugées pertinentes en regard de la question de recherche, c'est souvent parce que les chercheurs avaient utilisé des analyses univariées alors qu'une approche multivariée leur aurait mieux permis d'atteindre leurs objectifs de recherche. Les études multivariées utilisaient presque toujours des variables de contrôle pertinentes afin de limiter les sources possibles de confusion. Les autres études utilisaient des analyses descriptives pour explorer de nouveaux thèmes, afin de générer de nouvelles hypothèses et d'envisager des techniques plus sophistiquées dans le cadre de futures recherches. Les conclusions ont été jugées non pertinentes lorsqu'elles dépassaient la portée des analyses, notamment lorsque les auteurs raisonnaient en termes de causalité pour décrire une association. Un article a été considéré comme limité quant à son utilité pour le champ des pratiques et politiques généralement lorsqu'aucune application pratique de ses résultats n'avait été clairement présentée, mais non à cause d'une absence réelle d'application potentielle.

En ce qui concerne les associations entre variables, certains articles étudiaient les enjeux liés à la corroboration des mauvais traitements et à la prestation de services dans l'ensemble de l'échantillon de cycles de l'ECI ou dans des suréchantillons provinciaux, alors que d'autres articles offraient des analyses utilisant des sousgroupes d'enquêtes classés selon les caractéristiques des enfants et des familles ou selon les caractéristiques d'un mauvais traitement en particulier ou de son risque. Les analyses utilisaient les diverses catégories de maltraitance comme variables de prédiction de la prestation de services, du placement ou des problèmes de fonctionnement chez l'enfant ou de danger, mais pas de prédiction de la corroboration des mauvais traitements. Il est intéressant de noter que la présence de danger n'a pas été utilisée pour prédire la corroboration, bien qu'un danger observable utilisé comme preuve de mauvais traitements puisse sans doute faciliter pour les intervenants de la protection à l'enfance la corroboration d'allégations de maltraitance.

Notre examen a révélé l'existence de nouveaux champs de recherche utiles pour les politiques. Un article décrivant les pratiques de signalement des professeurs et la réponse à celles-ci des services de protection de l'enfance $^{23}$ illustre la présence de professionnels d'horizons variés impliqués dans la lutte contre la maltraitance à l'égard des enfants. On connait ainsi mieux les pratiques de signalement des différentes professions, un autre article traitant par exemple du processus de signalement de cas aux organismes de protection de l'enfance par les professionnels de la santé39. Dans la même veine, un article abordait les variables associées aux enquêtes menées conjointement par les services de police et par les intervenants de protection de l'enfance ${ }^{31}$.

Malgré la volonté de minimiser les modifications au questionnaire de l'ECI entre les cycles, certains changements ont été effectués afin de refléter des changements dans les pratiques elles-mêmes. Par exemple, le cycle de l'ECI 2008 a été modifié afin de recueillir de l'information sur les enquêtes pour risque ${ }^{3}$. L'un des articles a ainsi voulu dégager les conditions d'apparition d'un nouveau risque de maltraitance à partir d'incidents passés de mauvais traitements ${ }^{18}$.

L'utilisation des données de l'ECI a évolué au fil du temps : alors que dans l'examen précédent $^{7} 54 \%$ des analyse étaient multivariées, dans le présent examen c'était le cas de 62,5 \% d'entre elles. Cependant, malgré l'utilisation accrue de techniques multivariées, les objectifs de la plupart des études ne présentaient pas d'hypothèses allant dans une direction claire, ce qui avait pourtant été recommandé ${ }^{7}$.

L'examen précédent ${ }^{7}$ avait permis de constater que la violence physique était la catégorie de maltraitance la plus souvent étudiée et l'exposition à la violence conjugale la moins étudiée. L'examen présenté ici nous apprend que presque tous les articles fondés sur des analyses multivariées incluaient soit toutes les formes de maltraitance soit aucune à titre de variables indépendantes ou de contrôle. La constatation que plusieurs publications aient mis l'accent sur l'exposition à la violence conjugale, à la violence psychologique et à la négligence est importante pour les décideurs car la négligence et l'exposition à la violence conjugale étaient les première et deuxième formes corroborées de mauvais traitements dans l'ECI 2008 et la violence psychologique la quatrième ${ }^{3}$. Les trois articles centrés sur la négligence en ont cerné les facteurs de risque ${ }^{29,33}$, ont constaté une faible présence de danger ${ }^{29}$ et ont constaté également que les pères étaient plus souvent susceptibles d'être absents dans les cas de négligence ${ }^{28}$. Les trois articles centrés sur la violence psychologique ont noté que l'amélioration dans la clarté des définitions pour l'ECI 2008 avait permis de différencier occurrence et risque de violence psychologique ${ }^{34}$ et ils ont également prouvé que la violence psychologique est généralement associée à d'autres formes de maltraitance ${ }^{11}$ et que les cas corroborés de violence psychologique sont associés à des répercussions émotionnelles plus graves que celles constatées avec les autres formes de maltraitance $^{10}$. Les trois articles centrés sur l'exposition à la violence conjugale et décrivant les caractéristiques des cas d'exposition à la violence conjugale seulement, d'exposition à la violence conjugale et à d'autres mauvais traitements et enfin d'exposition à d'autres mauvais traitements seulement ${ }^{25}$ ont permis d'avancer que l'exposition à des sous-catégories différentes de violence conjugale peut conduire à des associations différentes au fonctionnement chez l'enfant ${ }^{6}$ et que les enquêtes sur l'exposition à la violence conjugale ou à des brutalités physiques seulement avaient des facteurs de risque moindres et étaient moins susceptibles de demeurer ouvertes que les autres enquêtes ${ }^{33}$.

Un autre thème d'importance sur les utilisations récentes des données de l'ECI est celui des enquêtes impliquant les enfants des Premières Nations. Cinq articles ont traité de thèmes spécifiquement liés aux enfants des Premières Nations, soit les facteurs ayant une incidence sur leur surreprésentation à l'étape de l'enquête ${ }^{30}$, les décisions liées au placement ${ }^{9,13,14}$ et les différences d'avec les rapports sur les enfants non autochtones ${ }^{32}$. 
Comparativement aux enquêtes visant les enfants non autochtones, celles visant les enfants des Premières Nations relevaient un pourcentage plus élevé pour chaque facteur de risque associé au pourvoyeur de soins ou au ménage (à l'exception des problèmes de santé) identifié par les intervenants ${ }^{30}$. Cette tendance pourrait justifier le besoin d'accrồtre la disponibilité des services d'aide aux familles dans les communautés des Premières Nations. Toutefois, le fait que les facteurs de risque aient été plus élevés pour ces familles peut relever d'un biais dans l'évaluation.

\section{Points forts et limites}

Notre examen comporte plusieurs points forts. Afin d'accroître l'exactitude de l'évaluation, tous les articles ont été examinés par au moins deux évaluateurs n'étant pas auteurs de ces articles. Nous avons utilisé le même outil normalisé d'évaluation de la qualité que celui du précédent examen sur l'utilisation des données de l'ECI ${ }^{7}$ afin que les conclusions soient comparables. Nous avons inclus à la fois des articles récents et des articles basés sur les données des suréchantillons, ce qui a permis d'accroître notre éventail d'études.

Notre examen présente également des limites. Il n'inclut aucun chapitre d'ouvrage, aucune thèse et aucun mémoire, aucun rapport gouvernemental ou d'organisme et aucun manuscrit non publié. Il est possible qu'en excluant ces sources nous ayons échoué à témoigner de l'envergure et de la profondeur de la recherche utilisant les données de l'ECI et celles du suréchantillon de l'ECI. Nous avons toutefois décidé d'exclure ces sources car elles ne sont généralement pas soumises à une évaluation par des pairs. Malgré l'existence de suréchantillons pour la Colombie-Britannique, l'Alberta, la Saskatchewan et les Territoires du Nord-Ouest, aucune analyse découlant de ces données n’a été, à notre connaissance, publiée dans une revue à comité de lecture. Les demandes de données pour ces suréchantillons ne passant pas nécessairement par l'Agence de la santé publique du Canada, il est également possible que certains articles nous aient échappé. De plus, étant donné que nous avons seulement inclus les articles publiés, nos conclusions peuvent souffrir d'un biais de publication (c'est-à-dire les corrélations entre variables statistiquement significatives peuvent avoir été surreprésentées parce que les associations non significatives ont moins de chance d'être publiées). Notons cependant à cet égard que la plupart des articles inclus présentaient également les résultats pour les associations non significatives entre variables. Nous avons inclus ces résultats dans le tableau 3 afin d'illustrer l'absence de liens entre certaines variables indépendantes et dépendantes.

Nos conclusions sont également dépendantes des limites relatives à la nature des données de l'ECI. Certaines d'entre elles ont été soulignées lors de l'examen des articles, en particulier les variations saisonnières, l'absence de vérification indépendante des données et l'utilisation d'informateurs par procuration. De plus, les données de l'ECI n'incluaient que les enfants dont la situation avait fait l'objet d'un signalement auprès d'un organisme d'aide à l'enfance ou d'une enquête auprès de ce dernier. Par conséquent, le biais de sélection peut avoir une incidence sur la population d'enfants considérée dans l'ECI, étant donné que les enfants ayant déjà subi de la maltraitance ou de la négligence (par exemple les enfants provenant de familles à faible revenu) sont davantage susceptibles de faire l'objet d'un rapport de l'aide à l'enfance que les autres enfants ${ }^{3}$.

De plus, la validité des critères associés aux variables des données de l'ECI est variable. Par exemple, les intervenants en protection de l'enfance qui enquêtent sur les cas de maltraitance ou d'allégation de maltraitance peuvent être davantage qualifiés pour l'évaluation des caractéristiques des mauvais traitements que pour celle des variables associées aux problèmes de fonctionnement chez l'enfant et le pourvoyeur de soins, puisque leur principal objectif est justement la caractérisation du mauvais traitement. Comme nous l'avons déjà mentionné ${ }^{40}$, les problèmes de fonctionnement chez l'enfant peuvent être sous-estimés car ils sont évalués au moyen d'une liste de vérification des problèmes connus ou pressentis par les intervenants en protection de l'enfance plutôt qu'au moyen d'une évaluation systématique normalisée, ce qui est impossible dans le cadre de l'étude. De plus, tous les éléments relatifs au fonctionnement chez l'enfant ne sont pas pertinents pour tous les groupes d'âge. Les études utilisant tout l'éventail des groupes d'âge des enfants ayant fait l'objet d'une enquête auraient pu, pour tenir compte de ce paramètre, être limitées aux âges des enfants qu'elles incluaient dans l'analyse, ce qui n'était pas le cas pour la plupart d'entre elles.

Enfin, les variables ont été mesurées au moment de l'enquête, ce qui fait qu'il est impossible de savoir si le mauvais traitement a eu lieu avant les problèmes de fonctionnement chez l'enfant, et par conséquent aucun lien de causalité n'a pu être établi. Ces limites sont importantes pour ceux qui utilisent ces connaissances comme pour les chercheurs eux-mêmes. Ces derniers pourraient tenter de voir comment améliorer la qualité des données en tenant compte des limites de l'étude.

\section{Conclusion}

Notre examen a décrit les évolutions dans l'usage des données de l'ECI et de ses suréchantillons pour répondre aux questions touchant la corroboration, le placement, la prestation de services et l'incidence des mauvais traitements sur le fonctionnement chez l'enfant. Il est évident que plusieurs facteurs influent sur ces résultats. Dans leurs analyses les plus récentes, les chercheurs se sont concentrés sur les catégories de maltraitance insuffisamment étudiées jusqu'ici (exposition à la violence conjugale, négligence et violence psychologique) et ont étudié les facteurs spécifiques touchant les enfants des Premières Nations ${ }^{7}$. Cet examen a révélé l'existence de nouveaux champs de recherche utiles pour les politiques. Il a également établi que, de manière générale, les données des suréchantillons de l'ECI ont été sous-utilisées dans la littérature évaluée par les pairs. Les chercheurs utilisant les données de l'ECI vont pouvoir tirer profit de ces résultats, car les erreurs fréquentes ont été relevées. La synthèse des conclusions de recherche peut aider les chercheurs à cerner les thèmes non étudiés de l'ECI. 
Dans le but d'atteindre les objectifs de recherche proposés, les études utilisant les données de l'ECI et de ses suréchantillons devraient continuer à utiliser des méthodes de modélisation statistique sophistiquées afin de tirer profit au maximum de l'information disponible.

\section{Remerciements}

Les auteurs aimeraient remercier les $\mathrm{D}^{\text {res }}$ Wendy Hovdestad et Anne-Marie Ugnat pour leur aide dans l'évaluation de certains articles inclus dans l'analyse présentée ici.

\section{Références}

1. Trocmé N, MacLaurin B, Fallon B et collab. Étude canadienne sur l'incidence des signalements de cas de violence et de négligence envers les enfants (EIC) : Rapport final. Ottawa (Ont.) : ministère des Travaux publics et des Services gouvernementaux Canada; 2001.

2. Trocmé N, Fallon B, MacLaurin B et collab. Étude canadienne sur l'incidence des signalements de cas de violence et de négligence envers les enfants - 2003. Ottawa (Ont.) : ministère des Travaux publics et des Services gouvernementaux Canada; 2005.

3. Agence de la santé publique du Canada. Étude canadienne sur l'incidence des signalements de cas de violence et de négligence envers les enfants : Données principales. Ottawa (Ont.) : Agence de la santé publique du Canada; 2010.

4. Tonmyr L, Jack SM, Brooks S, Williams G, Campeau A, Dudding P. Utilisation de l'Étude canadienne sur l'incidence des signalements de cas de violence et de négligence envers les enfants (ECI) par les organismes de protection de l'enfance en Ontario. Maladies chroniques et blessures au Canada. 2012;33(1):33-43.

5. Tonmyr L, Martin WK. How has child maltreatment surveillance data been used in Canada?. Health Res Policy Syst. 2014; 12(1):65:1-9.

6. Gonzalez A, MacMillan H, Tanaka M, Jack SM, Tonmyr L. Subtypes of exposure to intimate partner violence within a Canadian child welfare sample: Associated risks and child maladjustment. Child Abuse Negl. 2014;38:1934-1944.
7. Tonmyr L, Ouimet $\mathrm{C}$, Ugnat AM. A review of findings from the Canadian Incidence Study of Reported Child Abuse and Neglect (CIS). Can J Public Health. 2012;103(2):103-112.

8. Afifi TO, Taillieu T, Cheung K, Laurence YK, Tonmyr L, Sareen J. Substantiated reports of child maltreatment from the Canadian Incidence Study of Reported Child Abuse and Neglect (CIS) 2008: examining child and household characteristics and child functional impairment. Can J Psychiatry. 2015;60(7):315-323.

9. Chabot M, Fallon B, Tonmyr L, MacLaurin B, Fluke J, Blackstock C. Exploring alternate specifications to explain agency-level effects in placement decisions regarding aboriginal children: further analysis of the Canadian Incidence Study of Reported Child Abuse and Neglect Part B. Child Abuse Negl. 2013; 37:61-76.

10. Chamberland C, Fallon B, Black T, Trocmé $\mathrm{N}$, Chabot M. Correlates of substantiated emotional maltreatment in the second Canadian Incidence Study. J Fam Viol. 2012;27:201-213.

11. Chamberland $C$, Laporte L, Lavergne $C$ et collab. Psychological maltreatment of children reported to youth protection services. J Emotional Abuse. 2005;5:65-94.

12. Clément M-E, Chamberland C, Tourigny $M$, Mayer M. Taxinomie des besoins des enfants dont les mauvais traitements ou les troubles de comportement ont été jugés fondés par la direction de la protection de la jeunesse. Child Abuse and Neglect. 2009; 33:750-765.

13. Fallon B, Chabot M, Fluke J, Blackstock C, MacLaurin B, Tonmyr L. Placement decisions and disparities among Aboriginal children: further analysis of the Canadian Incidence Study of Reported Child Abuse and Neglect Part A: comparisons of the 1998 and 2003 surveys. Child Abuse Negl. 2013;37:47-60.

14. Fallon B, Chabot M, Fluke J, Blackstock C, Sinha V, Allan K, MacLaurin B. Exploring alternate specifications to explain agencylevel effects in placement decisions regarding Aboriginal children: further analysis of the Canadian Incidence Study of Reported Child Abuse and Neglect Part C. Child Abuse Negl. 2015 [sous presse].
15. Fallon B, Ma J, Black T, Wekerle C. Characteristics of young parents investigated and opened for ongoing services in child welfare. Int $\mathrm{J}$ Ment Health Addiction. 2011;9:365-381.

16. Fallon B, Ma J, Allan K, Pillhofer M, Trocmé $\mathrm{N}$, Jud A. Opportunities for prevention and intervention with young children: lessons from the Canadian Incidence Study of Reported Child Abuse and Neglect. Child Adolesc Psychiatry Ment Health. 2013;7:1-13.

17. Fallon B, Ma J, Allan K, Trocmé N, Jud A. Child maltreatment-related investigations inving infants: opportunities for resilience? Int J Child Adolesc Resil. 2013;1:35-47.

18. Fallon B, Trocmé N, MacLaurin B, Sinha V, Black T. Untangling risk of maltreatment from events of maltreatment: An analysis of the 2008 Canadian Incidence Study of Reported Child Abuse and Neglect (CIS-2008). Int J Ment Health Addiction. 2011;9:460-479.

19. Fast E, Trocmé N, Fallon B, Ma J. A troubled group? Adolescents in a Canadian child welfare sample. Child Youth Serv Rev. 2014; 46:47-54.

20. Feldman M, McConnell D, Aunos M. Parental cognitive impairment, mental health, and child outcomes in a child protection population. J Ment Health Res Intellect Disabil. 2012;5(1):66-90.

21. Hovdestad W, Shields M, Williams G, Tonmyr L. Vulnérabilité des familles dirigées par une mère adolescente ou jeune adulte faisant l'objet d'une enquête menée par un service de protection de l'enfance au Canada. Promotion de la santé et prévention des maladies chroniques. 2015;35(8/9):152-60.

22. Jud A, Fallon B, Trocmé N. Who gets services and who does not? Multi-level approach to the decision for ongoing child welfare or referral to specialized services. Child Youth Serv Rev. 2012;34:983-988.

23. King CB, Scott KL. Why are suspected cases of child maltreatment referred by educators so often unsubstantiated? Child Abuse Negl. 2014;38:1-10

24. Lee B, Rha W, Fallon B. Physical abuse among Asian families in the Canadian child welfare system. J Aggress Maltreat Trauma. 2014;23:532-551. 
25. Lefebvre R, Van Wert M, Black T, Fallon B, Trocmé N. A profile of exposure to intimate partner violence investigations in the Canadian child welfare system: an examination using the 2008 Canadian Incidence Study of Reported Child Abuse and Neglect (CIS-2008). Int J Child Adolesc Resil. 2013;1(1):60-73.

26. Ma J, Van Wert M, Lee B, Fallon B, Trocmé N. Non-English/non-French speaking caregivers involved with the Canadian child welfare system: Findings from the Canadian Incidence Study of Reported Child Abuse and Neglect (CIS-2008). Int J Child Adolesc Resil. 2013; 1(1):23-34.

27. Marcotte J, Cloutier R, Tourigny M et collab. Mauvais traitements ou troubles de comportement : Étude des variables qui distinguent l'appartenance à l'un ou l'autre de ces problématiques. Intervention. 2003;119: 58-70.

28. Mayer M, Dufour S, Lavergne C, Girard M, Trocmé N. Structures familiales, paternité et négligence : des réalités à revisiter. Revue de psychoéducation. 2006;35(1):155-176.

29. Ruiz-Casares M, Trocmé N, Fallon B. Supervisory neglect and risk of harm. Evidence from the Canadian child welfare system. Child Abuse Negl. 2012;36:471-480.

30. Sinha V, Trocmé N, Fallon B, MacLaurin B. Understanding the investigation-stage overrepresentation of First Nations children in the child welfare system: an analysis of the First Nations component of the Canadian Incidence Study of Reported Child Abuse and Neglect 2008. Child Abuse Negl. 2013;37:821-831.

31. Tonmyr L, Gonzalez A. Corrélats des enquêtes conjointes des services de protection de l'enfance et des services de police sur les abus sexuels d'enfants : résultats de l'Étude canadienne sur l'incidence des signalements de cas de violence et de négligence envers les enfants 2008. Promotion de la santé et prévention des maladies chroniques. 2015;35(8/9):138-46.

32. Tourigny M, Domond P, Trocmé N, Sioui B, Baril K. Incidence of maltreatment of Aboriginal children reported to youth protection in Quebec: intercultural comparisons. First Peoples Child Family Rev. 2007;3(3):103-119.
33. Trocmé N, Fallon B, Sinha V, Van Wert M, Kozlowski A, Maclaurin B. Differentiating between child protection and family support in the Canadian child welfare system's response to intimate partner violence, corporal punishment, and child neglect. Int $\mathrm{J}$ Psychol. 2013;48(2):128-140.

34. Trocmé N, Fallon B, MacLaurin et collabShifting definitions of emotional maltreatment: an analysis child welfare investigation laws and practices in Canada. Child Abuse Negl. 2011;35:831-840.

35. Trocmé N, Fallon B, MacLaurin B, Neves T. What is driving increasing child welfare caseloads in Ontario? Analysis of the 1993 and 1998 Ontario Incidence Studies. Child Welfare. 2005;84(3):341-362.

36. Trocmé N, Kyte A, Sinha V, Fallon B. Urgent protection versus chronic need: clarifying the dual mandate of child welfare services across Canada. Soc Sci. 2014;3:483-498.

37. Trocmé N, McPhee D, Tam KK. Child abuse and neglect in Ontario: uncidence and characteristics. Child Welfare. 1995;74(3):563586.

38. Van Wert M, Ma J, Lefebvre R, Fallon B. An examination of delinquency in a national Canadian sample of child maltreatmentrelated investigations. Int $\mathrm{J}$ Child Adolesc Resil. 2013;1(1):48-59.

39. Tonmyr L, Li A, Williams G, Scott D, Jack S. Patterns of reporting to child protection services in Canada by healthcare and nonhealthcare professionals. Paediatr Child Health. 2010;15(8):e25-e32.

40. Nasserie T. Éléments relatifs au fonctionnement de l'enfant dans l'Étude canadienne sur l'incidence des signalements de cas de violence et de négligence envers les enfants (ECI). Ottawa (Ont.) : Agence de la santé publique du Canada; 2014. 